\title{
BioRel: towards large-scale biomedical relation extraction
}

\author{
Rui Xing, Jie Luo* ${ }^{*}$ and Tengwei Song
}

From Biological Ontologies and Knowledge bases workshop 2019 San Diego, CA, USA. 18-21 November 2019

*Correspondence:
luojie@nlsde.buaa.edu.cn
State Key Laboratory
of Software Development
Environment, School
of Computer Science
and Engineering, Beihang
University, No. 37 Xueyuan
Road, Haidian District,
Beijing 100191, China

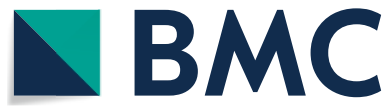

\begin{abstract}
Background: Although biomedical publications and literature are growing rapidly, there still lacks structured knowledge that can be easily processed by computer programs. In order to extract such knowledge from plain text and transform them into structural form, the relation extraction problem becomes an important issue. Datasets play a critical role in the development of relation extraction methods. However, existing relation extraction datasets in biomedical domain are mainly human-annotated, whose scales are usually limited due to their labor-intensive and time-consuming nature.

Results: We construct BioRel, a large-scale dataset for biomedical relation extraction problem, by using Unified Medical Language System as knowledge base and Medline as corpus. We first identify mentions of entities in sentences of Medline and link them to Unified Medical Language System with Metamap. Then, we assign each sentence a relation label by using distant supervision. Finally, we adapt the state-of-the-art deep learning and statistical machine learning methods as baseline models and conduct comprehensive experiments on the BioRel dataset.
\end{abstract}

Conclusions: Based on the extensive experimental results, we have shown that BioRel is a suitable large-scale datasets for biomedical relation extraction, which provides both reasonable baseline performance and many remaining challenges for both deep learning and statistical methods.

Keywords: Distant supervision, Relation extraction, Information extraction, Medline

\section{Backgrounds}

In recent years, we have witnessed a rapid growth in biomedical literature. Cohen and Hunter [1] provide an explanation on why the growth in PubMed and Medline publications is phenomenal. However, all the biomedical knowledge in these publications is expressed in the form of unstructured text, which cannot be easily utilized by computer programs. In fact, it is also very hard to manually transform all these knowledge in publications into structured form due to the large quantity of publications. Hence, automated text processing methods for transforming knowledge in text form into machine

(c) The Author(s) 2020. Open Access This article is licensed under a Creative Commons Attribution 4.0 International License, which permits use, sharing, adaptation, distribution and reproduction in any medium or format, as long as you give appropriate credit to the original author(s) and the source, provide a link to the Creative Commons licence, and indicate if changes were made. The images or other third party material in this article are included in the article's Creative Commons licence, unless indicated otherwise in a credit line to the material. If material is not included in the article's Creative Commons licence and your intended use is not permitted by statutory regulation or exceeds the permitted use, you will need to obtain permission directly from the copyright holder. To view a copy of this licence, visit http:// creativecommons.org/licenses/by/4.0/. The Creative Commons Public Domain Dedication waiver (http://creativecommons.org/publi cdomain/zero/1.0/) applies to the data made available in this article, unless otherwise stated in a credit line to the data. 
understandable format are getting more and more attention in biomedical science these days.

Relation extraction, a fundamental technique in Natural Language Processing (NLP), is very suitable to fulfill this task. It would be much easier for biomedical scientists to read and adjust new publications quickly by providing relations between certain entities, for example, possible treatment relation between ciprofloxacin and pyelonephritis. Plenty of recent studies in relation extraction adopted distant supervised paradigm [2]. Distant supervision aligns entity mentions in plain texts with those in knowledge bases to automatically generate relation instances. It assumes that if two entities have a certain relation in knowledge bases, then all sentences containing this entity pair will express that relation.

Previous studies adopted conventional statistical and graphical methods $[3,4]$ to detect relations. However, the main disadvantage is obvious in such pipeline since these traditional features explicitly derived from NLP tools can cause error propagation. As deep learning techniques $[5,6]$ have been widely studied and adopted, plenty of work applied deep neural network for distant supervised relation extraction. Zeng [7] proposed piecewise convolution neural network to build sentence representations and incorporated it into Multi-Instance Learning framework. Lin [8] expected to dynamically reduce the weights of those noisy instances and proposed selective attention over instances. Ji [9] developed a similar attention strategy together with entity descriptions to calculate weights over sentences. Liu [10] proposed a soft-label method to reduce the influence of entity-level noisy instances. Jat [11] used entity-based attention on wordlevel for efficient relation extraction. Since self-attention mechanism is proved to be effective and efficient, Du [12] started to use structured word-level self-attention and sentence-level attention mechanism to learn rich aspects of sentence representations.

Various relation extraction datasets have also been created in recent years. Doddington [13] introduced ACE 2004, Walker [14] built ACE 2005 dataset for relation classification, and Hendrick [15] proposed SemEval-2010 Task 8. All these datasets aimed at extracting relations in general domain such as news and web. In biomedical relation extraction, there are some widely used dataset such as Bacteria Biotope subtask (BB3), Seed Development subtask (SeeDev), Genia Event subtask (GE4) which is proposed in BioNLP 2016 Shared Task, BioNLP 2019 Shared Task, ${ }^{1}$ Drug-Drug Interaction (DDI) and Chemical Disease Relation (CDR). However, the main drawback is that these datasets are still manually labeled, requiring too much effort from linguists and biomedical experts, which also limits their scales. Besides, there are still some other problems which worth noticing in biomedical relation extraction. (1) Although these statistical approaches achieve promising results, they are still far from satisfactory. (2) Moreover, with the development of deep learning, the scale of human-annotated data is not large enough for deep learning model training and evaluation. (3) The total amount of training and testing instances is also limited in these datasets, which cannot reflect some relation aspects mentioned in the biomedical publications. All these raise the need for creating a large-scale and high quality biomedical dataset for distant supervised relation extraction.

\footnotetext{
${ }^{1}$ https://2019.bionlp-ost.org/.
} 
Table 1 Some examples in BioRel dataset

\begin{tabular}{|c|c|}
\hline Relation & Sentence \\
\hline Anatomic structure has location & $\begin{array}{l}\text { A brain mass and a spinal cord were identified in the cranial cavity } \\
\text { and the vertebral canal }\end{array}$ \\
\hline Therapeutic class of & $\begin{array}{l}\text { The histamine induced facilitation was blocked completely by cime- } \\
\text { tidine and antidepressant drugs imipramine and desipramine, } \\
\text { but remained unaffected in mice pretreated with mepyramine or } \\
\text { atropine }\end{array}$ \\
\hline Has physical part of anatomic structure & $\begin{array}{l}\text { In normal fibroblastoid cells } 30 \text { min after cultivation the cortical layer } \\
\text { would be well defined and demarcated from the adjacent cyto- } \\
\text { plasma, microfibrillae constituting it are parallel to one another and } \\
\text { perpendicular to the cell membrane }\end{array}$ \\
\hline May treat & $\begin{array}{l}\text { Treatment with oral ciprofloxacin should offer substantial cost savings } \\
\text { over a variety of parenteral antimicrobial regimens (e.g. aminoglyco- } \\
\text { side + beta-lactams) for difficult to treat infections such as chronic } \\
\text { pyelonephritis, osteomyelitis, and skin structure infections }\end{array}$ \\
\hline May be treated by & $\begin{array}{l}\text { The ventricular effective refractory period, as well as the vt cycle } \\
\text { length, increased after propranolol and was further prolonged after } \\
\text { the addition of a type i agent }\end{array}$ \\
\hline
\end{tabular}

Therefore in this extended version of our previous conference paper [16], we propose the Biomedical Relation Extraction Dataset (BioRel), a large-scale dataset for biomedical relation extraction. BioRel is constructed through distant supervision process, adopting Unified Medical Language System (UMLS) [17] as Knowledge Base (KB) and Medline as corpora resources. UMLS contains information of large amount of various biomedical entities and a wide range of relations. It is not only a rich knowledge base, but also a powerful tool for biomedical text processing. Medline is the U.S. National Library of Medicine (NLM) premier bibliographic database which contains more than 25 million references to journal articles in life sciences with a concentration on biomedicine. Medline is freely available on the Internet. Some selected instances and their entity and relation labels are shown in Table 1.

In order to assess the quality of our BioRel dataset, a variety of conventional statistical models and deep learning approaches are re-implemented and deployed as baselines for distant supervised relation extraction. Experiments on BioRel show that deep neural network (DNN) based approaches have better performance in comparison to statistical approaches, which demonstrates the capability of BioRel as a dataset for training deep neural network models. The differences of performances between models which are built to alleviate mislabeling problems are marginal, indicating that the mislabeling problem in BioRel is less serious in comparison to that in datasets of general domain such as NYT.

To summarize, the contributions of our work are as follows:

- We proposed a large-scale dataset for distant supervised biomedical relation extraction;

- We successfully adapt many state-of-the-art statistical and deep learning based approaches to BioRel;

- We conduct comprehensive evaluation of various baselines on our dataset, which indicates that BioRel has less noisy instances and is suitable for both deep learning 
and statistical based methods by providing reasonable baseline performance and many remaining challenges.

\section{Results}

Our experiments are intended to demonstrate and evaluate the quality of our dataset. In this section, comprehensive experiments are conducted to this end. We first introduce distant supervised relation extraction task formulation. Next, we describe overall experimental settings including word embeddings and parameter settings. And then we compare performance of several state-of-the-art methods on BioRel.

\section{Task formulation}

Multi-Instance-Learning (MIL) is a widely-used framework adopted by most stateof-the-art models in distant supervision. In MIL, the training set are separated into $n$ bags $\left\{\left\langle h_{1}, t_{1}\right\rangle,\left\langle h_{2}, t_{2}\right\rangle, \ldots,\left\langle h_{n}, t_{n}\right\rangle\right\}$, each of which contains $m$ sentences $\left\{s_{1}, s_{2}, \ldots, s_{m}\right\}$ mentioning same head entity $h_{i}$ and tail entity $t_{i}$. Each sentence consists of a sequence of $k$ words $\left\{x_{1}, x_{2}, \ldots, x_{k}\right\}$. For example, the following two instances containing the same entity pair in a bag, are sharing the same relation may be treated by. Instance 1 : "Three cases of acute myeloid leukaemia developing after treatment of renal disease with cyclophosphamide have been studied ". Instance 2: "Previous clinical trials have reported that cyclophosphamide can be used for the treatment of acute myeloid leukaemia." It's worth noting that the number of sentences $m$ is not always the same in each bag.

\section{Experimental settings}

For most the state-of-the-art neural network models achieving promising results, a cascade of commonly used approaches are adopted, which proved to be effective among various works $[8,9,11]$. To begin with, sentence representation $s_{i}$ is acquired using encoders on words $\left\{x_{1}, x_{2}, \ldots, x_{k}\right\}$, each of which is a word embedding. Then, bag representation $b_{i}=\left\langle h_{i}, t_{i}\right\rangle$ is produced using sentence representations within. For example, selective attention mechanism is first adopted in [8]. Next, fully connected network is trained as classifier for relation classification on bag level.

Word Embeddings Most previous work adopted word embeddings which are traditionally computed from a large corpus of unlabeled text, ignoring domain specific knowledge within. This information can potentially greatly improve the quality of word representation. Therefore in this work, we adopt BioWordVec [18] as word embeddings for all neural model baselines. BioWordVec is an open set of biomedical word embeddings that combines subword information from unlabeled biomedical text with a widely used biomedical controlled vocabulary called Medical Subject Headings (MeSH). The advantage of using BioWordVec is that it contains information in biomedical domain specific structured resources.

Position embeddings are initialized with Xavier for all baseline models. Word embeddings of blank words are initialized with zero while unknown words are initialized with the normal distribution of which the standard deviation is 0.05 . 
Table 2 Parameter settings

\begin{tabular}{lllllll}
\hline Settings & CNN & PCNN & GRU & MIML & MultiR & Mintz \\
\hline Batch size & 256 & 128 & 128 & - & - & - \\
Epoch & 20 & 20 & 20 & 10 & 15 & 15 \\
Learning rate & 0.4 & 0.2 & 0.3 & - & - & - \\
Word dim & 200 & 200 & 200 & - & - & - \\
Position dim & 10 & 10 & 10 & - & - & - \\
Sentence dim & 230 & 230 & 230 & - & - & - \\
Window size & 3 & 5 & - & - & - & - \\
Dropout & 0.5 & 0.5 & 0.3 & - & - & - \\
\hline
\end{tabular}

Parameter settings Cross-validation strategy is adopted to determine the parameters for our baseline models. And grid search is used to select learning rate $\lambda$ among $\{0.2,0.3,0.5\}$, sliding windows size among $\{3,5,7\}$, sentence embedding size among $\{100,200,300,400\}$ and batch size among $\{32,64,128,256\}$. Other parameters proved to have little effect on results. Our optimal parameter settings are shown in Table 2.

\section{Baseline models}

Mintz Mintz [2] first proposed distant supervision and considered it as a multi-class classification problem. In this work, standard lexical as well as syntactic features are utilized. And for each entity pair that appears in Freebase, all sentences containing this entity pair are collected and then used to train a relation classifier. Lexical features describe specific words between or surrounding an entity pair in the sentence, including the sequence of words between two entities, the part-of-speech tags of these words, a window of $k$ words to the head entity and another window of $k$ words to the tail entity.

MultiR Hoffmann proposed MultiR [19], a probabilistic, graphical model which takes positive and negative bags as input rather than single sentences. MultiR adpoted MultiInstance-Learning framework, which means that a bag will be labeled positive if it contains as least one positive example, otherwise it will be labeled negative if it contains no positive instance at all. MultiR is freely available and also adapted to our BioRel dataset in this paper.

MIMLRE Surdeanu [4] proposed a novel approach for multi-instance multi-label relation extraction. This approach built models for all instances sharing the same entity pair in the text, and also utilized a graphical model with latent variables to classify their relations. Instead of using original data published [4], we adapted the model to our BioRel dataset.

CNN Zeng [20] applied deep neural network to this task, which adopted Convolution Neural Networks (CNN) as encoders to extract sentence level features and used pre-trained word embeddings on large unlabeled corpus. This work proposed Positional Embeddings which proved to be effective. Positional Embeddings is defined as the combination of relative word distances between the current word to head entity $e_{1}$ and tail entity $e_{2}$. Zeng [20] randomly initializes position embedding matrices and then encodes these distances into vectors. This model is re implemented under MIL framework to fit our own task on BioRel. 

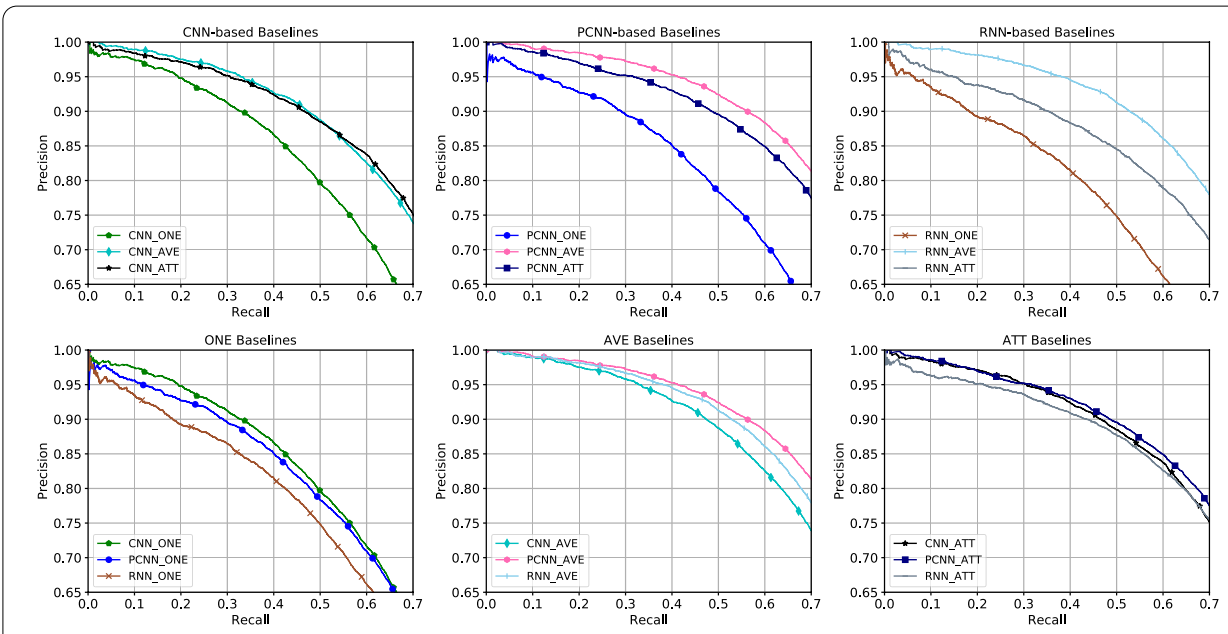

Fig. 1 Precision/recall curves of CNN, PCNN and GRU-based models

PCNN Zeng [7] proposed Piecewise Convolution Neural Networks (PCNN). This work treated distant supervised relation extraction as a multi-instance problem and also adopt $\mathrm{CNN}$ as sentence encoders. A great contribution of this work is piecewise max pooling technique. This method divides each sentence into three segments based on entity positions, enabling the model to capture structural information for relation extraction. Zeng also demonstrated that incorporating multi-instance learning can effectively solving the wrong label problem and further improve the performance.

PCNN+Selective Attention Lin [8] proposed a sentence-level attention-based approach for distant supervised relation extraction. The model embedded the sentence with $\mathrm{CNN}$ and built selective attention mechanism over multiple instances. Attention mechanism (ATT) dynamically adjusts weight $\alpha$ for each sentence. In this method, each sentence is first encoded into a vector representation, afterwards, the bag representation is calculated by taking an attention-weighted average of all the sentences in that bag. Comprehensive experiments under "only-one most likely sentence" (ONE) and "average vector over all instances" (AVE) environments are also conducted. In our work, we also follow these experiment environment settings (AVE, ATT, ONE) for better comparison.

RNN A large variety of work have been utilizing RNN-based models like LSTM [21] and GRU [22] for distant supervised relation extraction task [9, 11, 12, 23-25]. These are more capable of capturing long-distance semantic features compared to CNNbased models. In this work, GRU is adopted as a baseline model, because it is simpler and faster for model comparison on our large-scale BioRel dataset. Selective attention mechanism is also re-implemented and incorporated into all of previous neural-based baseline encoders. Additionally, "only-one" and "average" settings are also considered to examine the performance of the model and noise in BioRel.

\section{Performance and analysis}

Results of all neural network methods are shown in Fig. 1. Our best neural network baselines among "ONE,'AVE" and "ATT" settings with statistical baselines are shown in 
Table 3 P@N for distant supervised relation extraction models on BioRel

\begin{tabular}{llllllll}
\hline Model & P@4000 (\%) & P@8000 (\%) & P@12000 (\%) & P@16000 (\%) & Mean (\%) & F1 & AUC \\
\hline CNN+ONE & 93.38 & 84.91 & 75.00 & 65.69 & 79.75 & 0.66 & 0.70 \\
CNN+AVE & 94.00 & 90.95 & 81.58 & 71.97 & 85.30 & 0.72 & 0.79 \\
CNN+ATT & 96.40 & 90.59 & 82.35 & 72.31 & 85.41 & 0.72 & 0.78 \\
PCNN+ONE & 92.15 & 83.80 & 74.53 & 65.46 & 78.98 & 0.65 & 0.69 \\
PCNN+AVE & 96.57 & 93.60 & 85.74 & 75.39 & 88.12 & 0.76 & 0.82 \\
PCNN+ATT & 96.15 & 91.11 & 83.27 & 73.40 & 85.98 & 0.73 & 0.79 \\
RNN+ONE & 88.89 & 81.05 & 71.58 & 63.13 & 76.16 & 0.63 & 0.66 \\
RNN+AVE & 96.67 & 92.83 & 83.97 & 73.53 & 87.00 & 0.74 & 0.80 \\
RNN+ATT & 94.60 & 89.63 & 81.81 & 72.54 & 84.65 & 0.72 & 0.78 \\
Mintz & 79.79 & 67.08 & 56.93 & 49.23 & 63.25 & 0.49 & 0.45 \\
MultiR & 72.70 & 66.93 & 40.32 & 20.21 & 50.04 & 0.30 & 0.23 \\
MIML & 73.35 & 59.01 & 48.63 & 31.40 & 53.09 & 0.43 & 0.39 \\
\hline
\end{tabular}

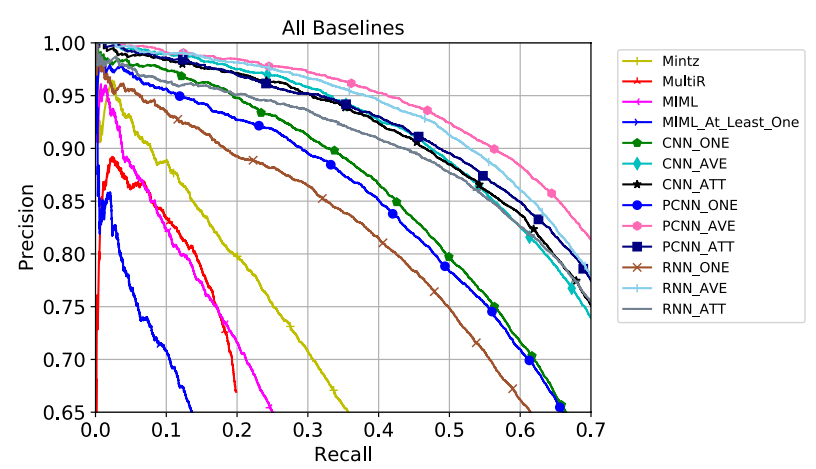

Fig. 2 Precision/recall curves of all baselines

Fig. 2. We also report the top precision results, F1-score and AUC of all baseline models in Table 3.

We have the following observations from above : (1) All methods achieve low performance under "ONE" setting, which demonstrates that only selecting "one most likely" sentence is less likely to obtain enough information, and failed to further obtain its bag representation. (2) Even when the recall is over 0.6, all neural baselines have reasonable precision over the entire range of recall, indicating that deep neural networks are effective in detecting various medical relations in our BioRel dataset. (3) Selective attention mechanism proved to be effective in general domain [8], and we expect models to perform better under "ATT" settings than under "AVE" settings. However, as can be seen from the Table 3, CNN and PCNN models have similar performance under "AVE" and "ATT" settings and GRU model performs even better under "AVE" than "ATT" settings. This indicates that attention mechanism for noise reduction tends to learn an average distribution of sentence representations within bags, which further proves that BioRel maintain high quality relation facts. (4) When recall is smaller than 0.05 , all models have reasonable precision among all baselines. While recall is higher, precision of feature-based models decrease sharply compared to neural network-based methods, the latter outperforms the former over the entire range of recall. It demonstrates that 
Table 4 Statistics of relation extraction datasets

\begin{tabular}{llccc}
\hline Dataset & Word & Sentence & Entity & Relation \\
\hline SemEval-2010 & $205 k$ & 10,717 & 21,434 & 9 \\
ACE 2003-2004 & $297 k$ & 12,783 & 46,108 & 24 \\
NYT & $21,457 k$ & 695,059 & 17,816 & 54 \\
BC5CDR & $282 k$ & 11,089 & 29,271 & 1 \\
BB3 & $34 k$ & 1394 & 2903 & 1 \\
SeeDev & $43 k$ & 1549 & 7082 & 22 \\
GE4 & $134 k$ & 5130 & 13,012 & 5 \\
i2b2 2010 & $91 k$ & 6310 & 8296 & 11 \\
BioRel & $26,166 k$ & 533,560 & 69,513 & 125 \\
\hline
\end{tabular}

human-designed features are limited and cannot concisely express semantic meaning of sentences.

\section{Discussion}

In this section, we analyze BioRel from different angles for a deeper understanding of our dataset.

\section{Data size}

Statistics of BioRel and some widely-used typical Relation Extraction datasets are shown in Table 3. These datasets include SemEval-2010 Task 8, ACE 2003-2004, NYT, BC5CDR, BB3, SeeDev, GE4, i2b2 2010. The first three datasets are used for generalpurpose relation extraction and the remaining for biomedical domain. From the table we find that BioRel is larger than existing datasets in both the total amount of words, entities and relations. We expect the large-scale BioRel dataset could facilitate deep learning methods in biomedical relation extraction.

Table 4 shows statistics of BioRel and some widely used representative relation extraction datasets.

\section{Named entity types}

BioRel contains a variety of entity types, including clinical drugs, pharmacologic substance, organic chemical, disease or syndrome, biologically active substance, molecular function, food, organ or tissue function and neoplastic process. We linked entities to their Concept Unique Identifiers (CUI) in UMLS and maintain their original expressions in sentences to provide challenges for models.

\section{Relation types}

BioRel consists of 124 labels corresponding to actual relations and a NA (Not A relation) label that indicates there is no relation between two entities. BioRel covers a wide range of relations, involving treatment, component of, side effect, metabolic mechanism, print names, etc. In particular, sentences with NA label account for nearly half of the dataset, which reflects the sparseness between entities of interest in real scenarios, i.e. assuming we randomly select two entities from certain biomedical publications, they are more likely to have no relation of interest. Therefore, we keep a large number of NA instances 
not only for making high quality model training, but also for making dataset realistic. Moreover, we take a certain relation attributes such as symmetric and asymmetric into consideration to provide further challenges for models. For example, we have both "may treat" and "may be treated by" relations in BioRel, as shown in Table 1.

\section{Sentence Instances}

The training set contains 534,406 sentences and was divided into 39,969 bags, validation set contains 218,669 sentences and 15,892 bags and testing set contains 114,515 sentences and 20,759 bags. Each bag contains sentences sharing the same head and tail entities. The average number of sentences in each bag is 13 in training set, 5 in validation set and 8 in testing set.

\section{Conclusions}

In this paper, we proposed a new large-scale dataset, BioRel, for distant supervised biomedical relation extraction. The dataset was created by aligning UMLS relations to Metamapped Medline Corpus. Relations which may cause false positive are filtered. Meanwhile, we manually kept strict order of relation between medical entities to provide challenges for models to detect exact relations. Comprehensive experiments conducted on the dataset show that the BioRel dataset is especially suitable for distant supervised biomedical relation extraction with the state-of-the-art neural network-based baseline models. Further analysis also suggested that BioRel may have fewer noisy sentences than the widely-used general-purpose NYT dataset.

BioRel enables several promising research directions in the future. It puts forward challenges to relation extraction models for further improving their performance. UMLS contains a large number of vocabularies, each of which focuses on a specific subdomain. We manually select NDFRT and NCI for relation extraction to facilitate automatic diagnosis. However, the process of BioRel construction can be replicated on other sub-domains as well. Therefore, other vocabularies like MeSH can be chosen freely to construct datasets that meet specific requirements of other researches.

Human annotation and evaluation are still essential. More accurate and precise results and analysis could be provided with the help of researchers and medical experts.

\section{Methods}

In this section, we describe the process of creating the dataset in detail. The whole procedure can be divided into the following three steps: data collection, entity recognition and linking, and distant supervision. In the data collection section, Medline and UMLS are used as the data source to provide corpora, entities and relations. Then, in entity recognition and linking part, MetaMap ${ }^{2}$ is adopted to recognize entities in sentences and link them to their unique identifiers. Finally, we obtain our datasets using distant supervision method which automatically aligns relations to Medline corpora.

\footnotetext{
${ }^{2}$ https://metamap.nlm.nih.gov/.
} 


\section{Data collection}

For the first step, we use Medline as the corpus and UMLS as our knowledge base. The data is made freely available on the Internet by the United States National Library of Medicine and can be accessed and searched by the search engine PubMed. ${ }^{3}$ Currently, Medline contains references to more than 25 million journal articles in life sciences with a concentration on biomedicine. In this work, sentences extracted from Medline documents are used to create data and perform extra experiments.

The Unified Medical Language System is a large biomedical knowledge base named Metathesaurus, which contains millions of biomedical entities and relations. MRREL is a subset of the Metathesaurus, which involves only binary relations between different biomedical entities. In this work, we only select two vocabularies as sources: NDFRT (National Drug File - Reference Terminology) and NCI (National Cancer Institute). NDFRT defines the relationship between drugs and diseases, while NCI contains information related to genes and cancer. We choose such selection for two reasons: (a) The number of instances for different relations is unevenly distributed in MRREL. Some relations contain thousands of instances while others only involve hundreds or fewer instances. The knowledge in Metathesaurus is organized into vocabularies related to certain topics. (b) These two vocabularies not only contain enough entities and relations, but also may further assist the development of automatic diagnosis and other technologies by connecting genes, cancer and treatment methods.

\section{Entity recognition and linking}

In order to identify entities in Medline corpus, we adopt MetaMap, a highly configurable program to discover UMLS Metathesaurus entities that referred to biomedical texts. MetaMap uses a knowledge-instensive method based on symbolic, natural-language processing and computational-linguistic techniques. The benefits of using MetaMap are three folds: First, MetaMap is freely available. Second, it has been widely adopted by many works. Finally, it can perform entity recognition and linking simultaneously.

\section{Feature extraction}

As shown in Table 5, in order to provide designed features for statistical relation extraction baselines, we extract lexical and syntactic features described in [2, 4, 19]. To take advantage of recent achievement, instead of using traditional statistical NLP tools, we adopt the deep neural network based StanfordNLP [26] tool for part-of-speech (POS) tagging and dependency parsing.

\section{Distant supervised annotation and filtering methods}

To introduce distant supervision in detail, we first traverse the entire Medline corpus and use Metamap to identify medical entities in sentences. Then, we enumerate all combinations of entity pairs in the sentence to create a candidate set. Similarly, relation triples in Metathesaurus are selected to form a knowledge base relation set. Finally, we remove some invalid instances that would affect the performance.

\footnotetext{
${ }^{3}$ https://www.ncbi.nlm.nih.gov/pubmed/.
} 


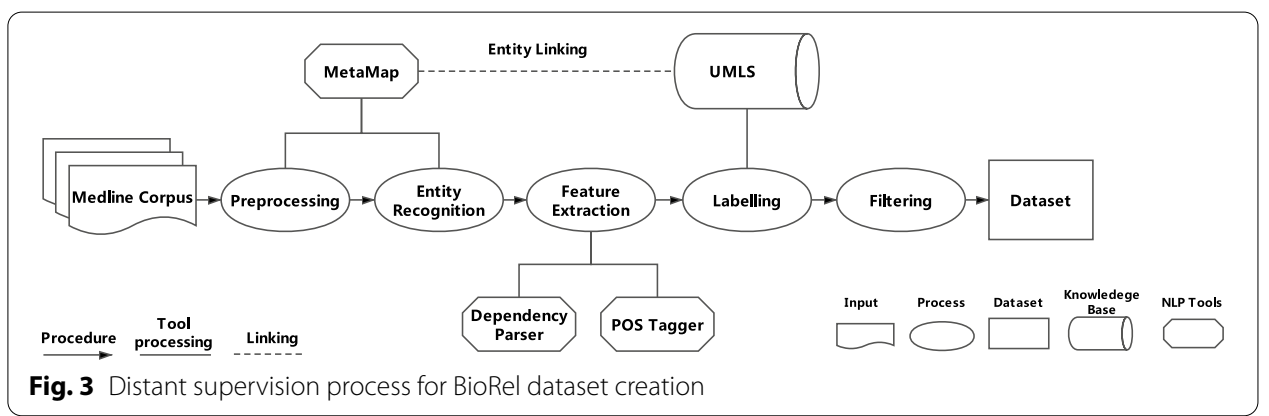

Table 5 features for traditional statistical baselines

\begin{tabular}{ll}
\hline Lexical & The sequence of words between the two entities \\
The part-of-speech of words between the two entities \\
A flag indicating which entity came first in the sentence \\
A window of k words to the left of the first entity and \\
their part-of-speech tags \\
A window of k words to the right of the second entity \\
and their part-of-speech tags \\
A dependency path between the two entities \\
Part-of-speech of words in dependency path \\
A'window' node that is not part of the dependency path
\end{tabular}

Distant Supervised annotation labels the relations between aligned entities in sentences according to the knowledge base. It assumes that if two entities have a relation in the knowledge base, all sentences containing the same two entities will express that relation. Formally, for each sentence in Medline containing head and tail entities $e_{1}$ and $e_{2}$, if there exists a relation triple $\left(e_{1}, e_{2}, r\right)$ in Metathesaurus indicating that $e_{1}$ and $e_{2}$ have the relation $r$, we use the label $r$ to denote the sentence containing this entity pair. For example, ciprofloxacin and pyelonephritis has the relation may treat in the knowledge base, then the sentence "Treatment with oral ciprofloxacin should offer substantial cost savings over a variety of parenteral antimicrobial regimens for difficult to treat infections such as chronic pyelonephritis, osteomyelitis, and skin structure infections." and "Ciprofloxacin has some effect on pyelonephritis." would both be labeled as may treat relation.

Although efficient and effective, distantly labeled data often contains false positive and false negative instances. We apply further filtering methods to remove some mislabeling data. First, sentences containing entity pairs with two mentions of the same entities are removed. According to Bobic [27], self-relations, i.e. relations between two different aliases of the same entities, are more likely to produce false positives. Second, lowfrequency entity pairs are discarded, because entity pairs which occur only a few times within millions of Medline sentences may cause bias in training process. The whole procedure is shown in Fig. 3.

\section{Evaluation methods}

In order to train and test relation extraction models, we divide the training, validation and testing set by randomly selecting instances of each relation from raw dataset. In 
each relation, $70 \%$ of instances are selected as training set, 15\% of instances are selected as validation set, and the remaining $15 \%$ are used as testing set. Following previous work $[2,8,10]$, we evaluate the baseline models on our dataset in the held-out evaluation which provides an approximate measure of precision without requiring expensive human evaluation. We draw precision-recall curves for all models and also report the Precision@N results.

\section{Abbreviations}

UMLS: Unified Medical Language System; CDR: Chemical Disease Relation; NYT: New York Times; NLM: National Library of Medicine; NDFRT: NationalDrug File - Reference Terminology; NCl: National Cancer Institute; CNN: Convolution Neural Network; PCNN: Piecewise Convolution Neural Network; RNN: Recurrent Neural Network; LSTM: Long Short Term Memory Networks; GRU: Gated Recurrent Units; P@N: Top N Precision; AUC: Area Under the receiver operating characteristic Curve; DDI: Drug-Drug Interaction.

\section{Acknowledgements}

We would like to thank all of the reviewers for their comments on the manuscript.

\section{About this supplement}

This article has been published as part of BMC Bioinformatics Volume 21 Supplement 16, 2020: Selected articles from the Biological Ontologies and Knowledge bases workshop 2019. The full contents of the supplement are available online at https://bmcbioinformatics.biomedcentral.com/articles/supplements/volume-21-supplement-16.

\section{Authors' contributions}

$R X$ and $J L$ conceived and designed the project. RX and TWS carried out the experiments. RX wrote the manuscript. JL provided guidance on the study and revised the manuscript. All authors read and approved of the final manuscript.

\section{Funding}

The funding bodies of this study had no role in the study design, data analysis and interpretation, or writing of the manuscript. The corresponding authors had full access to all the data in the study and had final responsibility for the decision to submit for publication. Publication costs are funded by National Natural Science Foundation of China (Grant No. 61690202) and State Key Laboratory of Software Development Environment (Grant No. SKLSDE-2017ZX-17).

\section{Availability of data and materials}

NYT-10 data used in this paper come from study [3], which can be downloaded from http://iesl.cs.umass.edu/riede l/ecml/. Medline corpora data can be downloaded from https://www.ncbi.nlm.nih.gov/pubmed/ and Metamap can be downloaded from https://metamap.nIm.nih.gov/. The BioRel dataset in this paper is available at https://bit.ly/biore I_dataset.

\section{Ethics approval and consent to participate}

Not applicable.

\section{Consent for publication}

Not applicable.

\section{Competing interests}

The authors declare that they have no competing interests.

Received: 16 November 2020 Accepted: 18 November 2020

Published: 16 December 2020

\section{References}

1. Cohen KB, Hunter L. Getting started in text mining. PLoS Comput Biol. 2008; https://doi.org/10.1371/journ al.pcbi.0040020.

2. Mintz M, Bills S, Snow R, Jurafsky D. Distant supervision for relation extraction without labeled data. In: ACL '09. Stroudsburg, PA, USA: Association for Computational Linguistics; 2009. p. 1003-11.

3. Riedel S, Yao L, McCallum A. Modeling relations and their mentions without labeled text. In: ECML PKDD'10. Berlin: Springer; 2010. p. 148-63.

4. Surdeanu M, Tibshirani J, Nallapati R, Manning CD. Multi-instance multi-label learning for relation extraction. Jeju Island: Association for Computational Linguistics; 2012. p. 455-65.

5. Bengio Y. Learning deep architectures for Al. Found Trends Mach Learn. 2009;2(1):1-127. https://doi. org/10.1561/2200000006.

6. LeCun Y, Bengio Y, Hinton GE. Deep learning. Nature. 2015;521(7553):436-44. https://doi.org/10.1038/nature14539.

7. Zeng D, Liu K, Chen Y, Zhao J. Distant supervision for relation extraction via piecewise convolutional neural networks. Lisbon: Association for Computational Linguistics; 2015. p. 1753-62.

8. Lin Y, Shen S, Liu Z, Luan H, Sun M. Neural relation extraction with selective attention over instances. Berlin: Association for Computational Linguistics; 2016. p. 2124-33. 
9. Ji G, Liu K, He S, Zhao J. Distant supervision for relation extraction with sentence-level attention and entity descriptions; 2017. p. 3060-6.

10. Liu T, Wang K, Chang B, Sui Z. A soft-label method for noise-tolerant distantly supervised relation extraction. Copenhagen: Association for Computational Linguistics; 2017. p. 1790-5.

11. Jat S, Khandelwal S, Talukdar P. Improving distantly supervised relation extraction using word and entity based attention. arXiv e-prints, 1804-06987; 2018. arXiv:1804.06987.

12. Du J, Han J, Way A, Wan D. Multi-level structured self-attentions for distantly supervised relation extraction. Brussels: Association for Computational Linguistics; 2018. p. 2216-25.

13. Doddington G, Mitchell A, Przybocki M, Ramshaw L, Strassel S, Weischedel R. The automatic content extraction (ACE) program - tasks, data, and evaluation. Lisbon, Portugal: European Language Resources Association (ELRA); 2004.

14. Walker C, Strassel S, Medero J, Maeda K. ACE 2005 multilingual training corpus; 2005.

15. Hendrickx I, Kim SN, Kozareva Z, Nakov P, Ó Séaghdha D, Padó S, Pennacchiotti M, Romano L, Szpakowicz S. SemEval-2010 Task 8: multi-way classification of semantic relations between pairs of nominals. Uppsala: Association for Computational Linguistics; 2010. p. 33-8.

16. Xing R, Luo J, Song T. Biorel: a large-scale dataset for biomedical relation extraction. In: 2019 IEEE international conference on bioinformatics and biomedicine (BIBM); 2019. p. 1801-8. https://doi.org/10.1109/BIBM47256.2019.89830 57.

17. Bodenreider $\mathrm{O}$. The unified medical language system (UMLS): integrating biomedical terminology. Nucleic Acids Res. 2004:32:267-70.

18. Yijia Z, Chen Q, Yang Z, Lin H, Lu Z. Biowordvec, improving biomedical word embeddings with subword information and mesh. Sci Data. 2019; https://doi.org/10.1038/s41597-019-0055-0.

19. Hoffmann R, Zhang C, Ling X, Zettlemoyer L, Weld DS. Knowledge-based weak supervision for information extraction of overlapping relations. Portland: Association for Computational Linguistics; 2011. p. 541-50.

20. Zeng D, Liu K, Lai S, Zhou G, Zhao J. Relation classification via convolutional deep neural network. Dublin: Dublin City University and Association for Computational Linguistics; 2014. p. 2335-44.

21. Hochreiter S, Schmidhuber J. Long short-term memory. Neural Comput. 1997;9:1735-80. https://doi.org/10.1162/ neco.1997.9.8.1735.

22. Cho K, van Merriënboer B, Gulcehre C, Bahdanau D, Bougares F, Schwenk H, Bengio Y. Learning phrase representations using RNN encoder-decoder for statistical machine translation. Doha: Association for Computational Linguistics; 2014. p. 1724-34. https://doi.org/10.3115/v1/D14-1179.

23. Zhang D, Wang D. Relation classification via recurrent neural network. CoRR; 2015. arXiv:1508.01006.

24. Zhou P, Shi W, Tian J, Qi Z, Li B, Hao H, Xu B. Attention-based bidirectional long short-term memory networks for relation classification. Berlin: Association for Computational Linguistics; 2016. p. 207-12. https://doi.org/10.18653/ v1/P16-2034.

25. Vashishth S, Joshi R, Prayaga SS, Bhattacharyya C, Talukdar P. RESIDE: improving distantly-supervised neural relation extraction using side information. Brussels: Association for Computational Linguistics; 2018. p. 1257-66.

26. Qi P, Dozat T, Zhang Y, Manning CD. Universal dependency parsing from scratch. Brussels: Association for Computational Linguistics; 2018. p. 160-70.

27. Bobić T, Klinger R, Thomas P, Hofmann-Apitius M. Improving distantly supervised extraction of drug-drug and protein-protein interactions. Avignon: Association for Computational Linguistics; 2012. p. 35-43.

\section{Publisher's Note}

Springer Nature remains neutral with regard to jurisdictional claims in published maps and institutional affiliations.

Ready to submit your research? Choose BMC and benefit from:

- fast, convenient online submission

- thorough peer review by experienced researchers in your field

- rapid publication on acceptance

- support for research data, including large and complex data types

- gold Open Access which fosters wider collaboration and increased citations

- maximum visibility for your research: over $100 \mathrm{M}$ website views per year

At BMC, research is always in progress.

Learn more biomedcentral.com/submissions 